\title{
Symptom clusters and quality of life in China patients with lung cancer undergoing chemotherapy
}

\author{
Dandan Wang, Jufang Fu
}

The First Affiliated Hospital of the Forth Military Medical University

\begin{abstract}
Objective: To explore the symptom clusters and quality of life in patients with lung cancer undergoing chemotherapy. Methods: A cross-sectional survey was completed with 183 patients from three public hospitals in Xi'an, China. Patients completed a demographic questionnaire, the Functional Assessment of Cancer Therapy-Lung Cancer (FACT-L) and the M. D. Anderson Symptom Inventory (MDASI-C). Factor analysis was used to identify symptom clusters based on the severity of patients' symptom experiences. The resulting clusters were correlated with quality-of-life measures.

Results: The QOL scores of lung cancer patients on the functioning subscale were the lowest $(9.70 \pm 5.30)$, while those of the family subscale were the highest (19.28 3 3.24). Three symptom clusters were identified: gastrointestinal, emotional and fatigue-related symptoms. There was a negative relationship between the symptom clusters and multiple dimensions of quality of life $(\mathrm{r}-0.178 \square-0.805, \mathrm{p}<0.05)$. Females, especially those women with low education level / chronic diseases, were experienced greater symptom distress than others.

Conclusions: The clusters had a negative relationship with QOL. Identifying symptom clusters helped clarify possible interrelationships which may lead to the establishment of more effective symptom management interventions for patients with lung cancer in order to improve the quality of life.
\end{abstract}

Keywords: symptom clusters, lung cancer, factor analysis, symptom management, quality of life African Health Sciences 2014;14(1): 49-55 http://dx.doi.org/10.4314/ahs.v14i1.8

\section{Introduction}

Lung cancer has become the leading cause of cancer deaths in mainland China, and more than 24600 people died of lung cancer in the year 2008[1]. Lung cancer was often diagnosed at an advanced stage with poor prognosis. Anecdotal data shows that $80 \%$ of lung cancer cases are diagnosed at stage 3 or 4 in the general hospitals in China. Patients with lung cancer experience a variety of distressing symptoms, many of which begin prior to diagnosis and continue throughout the course of the disease and its treatment, adversely affecting functional status and quality of life (QOL). Compared to other types of cancer, the distress associated with symptoms arising from lung cancer has been reported as the most intense[2]. Part of the symptom burden experienced by patients with lung cancer may be the

\section{Corresponding author:}

Jufang $\mathrm{Fu}$

Department of infection management

Xijing Hospital

The First Affiliated Hospital of the Forth

Military Medical University

No.15, Changle West Road, Xi'an 710032, People's Republic of China

E-mail: icanspeak@126.com result of the simultaneous occurrence of symptoms, also known as "clustering" of symptoms. The term"symptom clusters" has been defined as two or more interrelated symptoms that are present together, independent of other symptom clusters, and may or may not suggest a common etiology or underlying mechanism [3-5]. Research has provided evidence that, together, multiple symptoms may have more negative effects on patients' quality of life than the occurrence of single symptoms [6,7].

Most research on symptoms has been directed towards a single symptom, such as pain or fatigue, or symptoms that were correlated with a single symptom. However, the symptoms generally occurred at the same time and there were synergic relationships among them that resulted in negative impact on QOL[8]. Thus, the exploration of the impact of simultaneous symptoms on QOL domains in lung cancer patients, and the understanding of symptom synergism, became important for successful achievement of cancer treatment. Establishing the best strategies to reduce the impact of cancer and treatment-related symptoms, and to improve QOL, are important. Appropriate symptom control, considering symptom interactions, should be a priority for patients with lung cancer. In addition, effective symptom management might lessen or negate these effects and improve lung cancer patients' quality of life. 
Therefore, the objectives of this study were to (1) describe symptom experienced by patients with lung cancer undergoing chemotherapy; (2) explore whether multiple symptoms were clustered into groups of symptoms; (3) examine whether symptom clusters were related to demographic, health characteristics; (4) identify the impact of symptom clusters on QOL dimensions

Various theoretical frameworks were available to guide our understanding of symptom clusters. We used the theory of unpleasant symptoms (TOUS), which has been utilized frequently in symptom cluster research [9-12]. TOUS has three main reciprocal components: symptoms, influential factors, and performance. According to the theory, each symptom has four dimensions: intensity, timing, level of distress perceived and quality. The symptoms of the effecting factor include physiological, psychological, and situational antecedents. Performance is the consequence of the symptom experience, which covered functional and cognitive activities. This model provides a theoretical framework for research on symptom clusters by indentifying multiplicative effects of multiple concurrent symptoms. In the theory of unpleasant symptoms, Lenz[13] proposed that symptom clusters have a resultant effect on important patient outcomes, such as QOL.

\section{Methods}

\section{Design}

A cross-sectional study design was used with a convenient sample of patients from three public hospitals in Xi'an from January to June of 2012.

\section{Sample and Settings}

The research criteria for the sample was determined to reflect the following conditions: (1) receiving chemotherapy for lung cancer at any stage of their chemotherapy, and any cycle; (2) 18 years of age or older; (3) able to understand and speak Chinese, and (4) absence of cognitive impairment.

A total of 200 patients were approached and invited to participate. Among them, 183 patients responded to the study invitation. Seventeen patients $(8.5 \%)$ were too sick to complete the questionnaires, or were prevented from participating by a family member.

\section{Procedures}

After the hospital ethics committee approved the study, eligible patients were screened and those meeting the selection criteria were invited to participate in the study. The research assistant contacted all patients and provided a verbal explanation of study. All patients were informed that their participation was voluntary, they would remain anonymous, they could withdraw from the study at any time without penalty, and all information would be kept confidential. After obtaining consent, all participants completed the MDASI-C, FACT-L and Demographic and Medical questionnaires. Research assistants were available on site during the administration of the questionnaires and provided explanation. The participant's demographic and clinical data was obtained through personal interview and the review of their medical records. The time required to complete the questionnaire was approximately $20 \square 30$ minutes.

\section{Instrument}

The study instruments included a demographic and clinical questionnaire, the Functional Assessment of Cancer Therapy-Lung Cancer (FACT-L) and the M. D. Anderson Symptom Inventory-the Chinese version $\square$ MDASI-C $\square$.

\section{The demographic and clinical questionnaire}

The demographic information included the gender, age, family monthly income, type of medical insurance, marital status, educational level, religious affiliation and employment status. The clinical information were about the type of cancer, extent of disease, presence of metastatic disease, and whether there was coexistence with other chronic diseases .

\section{Chinese Version of the M. D. Anderson Symptom Inventory} (MDASI-C)

The MDASI-T was used to measure the severity of symptoms and the degree to which they interfered with daily life. The MDASI had been established as a valid and reliable tool for assessing cancer-related symptoms, regardless of therapy or specific diagnosis. It contained 13 core symptom severity items (that is: fatigue, sleep disturbance, pain, drowsiness, lack of appetite, nausea, vomiting, shortness of breath, numbness, difficulty remembering, dry mouth, distress, and sadness) which could explain $64 \%$ of the variance in symptom interference, and six symptom interference items (that is: general activity, mood, work, relations with other people, walking, and enjoyment of life). A severity composite score was computed by averaging the scores for the 13 severity items. An interference composite score was computed by averaging the scores 
for the six symptom interference items. The MDASI was rated from 0 (symptom has not been present) to 10 (the symptom was as bad as I could imagine it to be) for each item. Internal consistencies of the symptom severity and the symptom in Chinese version were 0.82 $\square$ 0.94. Most patients completed the form in 5 minutes.

\section{The Functional Assessment of Cancer Therapy-Lung Cancer} (FACT-L)

Quality of life was measured using the Chinese version of the Functional Assessment of Cancer Therapy V Lung cancer (FACT-L 4.0) developed specifically for patients with lung cancers[14]. Functional Assessment of Cancer Therapy V Lung cancer contained the original FACT-General scales that included a 27 -item compilation of general questions divided into 4 primary QOL domains: physical (7 items), social/family(7 items), emotional (6 items), and functional well-being(7 items). The additional 9 items were used to assess disease-specific issues in patients with lung cancer[14]. The subjects circle numbers on a scale were from 0 (not at all) to 4 (very much) indicating their reaction to each statement. Higher scores for the scales and subscales indicated better QOL. The possible total scores ranged from 0 to 144. In this study, internal consistency of FACT-L (Chinese version 4.0) was $0.56 \square 0.82$, whereas the test-retest reliability was $0.76 \square 0.82[15]$.

\section{Statistical Analysis}

Data were stored and analyzed using SPSS version 13.0. Frequency distributions and descriptive statistics were used to analyze the demographic, socioeconomic, and clinical data, the scores on each symptom and QOL questionnaires (the subscales and the global QOL scores).

Exploratory factor analysis with principal axis factoring was used to identify symptom clusters. Varimax rotation was used to maximize the variance of the loadings within each component while assuming the independence of the component structure Spearman correlations was utilized to examine correlations between symptom clusters and QOL: total score of all symptoms of each symptom cluster and global QOL score, score of each dimension of QOL.

\section{Results}

Demographic characteristics and clinical information

A total of 183 participants undergoing chemotherapy were recruited. Most of the participants $(63.9 \%)$ were male. The mean age was 58.25 years $(S D=10.31$, range $=32 \square 84)$. A large number of participants $(n=174)$ were married (95.1\%); 66.7\% were unemployed, 56.3\% were educated 9 years or less, and $90 \%$ had no religious beliefs, a typical characteristic of Chinese culture.

The diagnosis of non-small-cell lung cancer (NSCLC) comprised $80.9 \%$ and $31.2 \%$ for smallcell lung cancer (SCLC). Patients with stage IV nonsmall-cell lung cancer (NSCLC) accounted for 38.8\%, while those with stage III non-small-cell lung cancer (NSCLC) totaled 22.4\%. Patients with extensive-stage small-cell lung cancer (SCLC) comprised only 6.0\% of the sample. More details of demographic and clinical data were presented in Table 1.

Table 1 Demographic characteristics and clinical information

\begin{tabular}{|c|c|c|c|c|c|c|}
\hline Variable & $\mathrm{n}$ & $\square \%$ & $\mathrm{M}(\mathrm{SD})$ & Variable & $\mathrm{n}$ & $\square \%$ \\
\hline Age & & & $\square 58.25 \pm 10.31 \square$ & Diagnosis & & \\
\hline Gender & & & & Small cell lung cancer & 35 & $19.1 \%$ \\
\hline Male & 117 & $63.9 \%$ & & Non-small cell lung cancer & 148 & $80.9 \%$ \\
\hline Female & 66 & $36.1 \%$ & & Stage of disease & & \\
\hline Marital status & & & & Non-small cell lung cancer II & 36 & $19.7 \%$ \\
\hline $\begin{array}{l}\text { Singe } \\
\text { Married } \\
\text { Divorced }\end{array}$ & $\begin{array}{l}4 \\
174 \\
5\end{array}$ & $\begin{array}{l}2.2 \% \\
95.1 \% \\
2.7 \%\end{array}$ & & $\begin{array}{l}\text { Non-small cell lung cancer III } \\
\text { Non-small cell lung cancer IV } \\
\text { Limited-stage small cell lung cancer }\end{array}$ & $\begin{array}{l}41 \\
71 \\
24\end{array}$ & $\begin{array}{l}22.4 \% \\
38.8 \% \\
13.1 \%\end{array}$ \\
\hline Education & & & & Extensive-stage small cell lung cancer & 18 & $9.8 \%$ \\
\hline $\begin{array}{l}\text { Primary school } \\
\text { Junior school } \\
\text { Senior school } \\
\text { College/university }\end{array}$ & $\begin{array}{l}53 \\
50 \\
44 \\
36\end{array}$ & $\begin{array}{l}29.0 \% \\
27.3 \% \\
24.0 \% \\
19.7 \%\end{array}$ & & $\begin{array}{l}\text { Family monthly income } \\
\text { Less than } 1,000 \\
1,000 \square 2,000 \\
2,000 \square 3,000\end{array}$ & $\begin{array}{l}79 \\
40 \\
33\end{array}$ & $\begin{array}{l}43.2 \% \\
21.9 \% \\
18.0 \%\end{array}$ \\
\hline Occupation & & & & More than 3,000 with other chronic & 31 & $16.9 \%$ \\
\hline No & 122 & $66.7 \%$ & & $\begin{array}{l}\text { diseases } \\
\text { Yes }\end{array}$ & 53 & $29.0 \%$ \\
\hline Yes & 61 & $33.3 \%$ & & No & 130 & $71.0 \%$ \\
\hline Medical insurance & & & & & & \\
\hline No & 10 & $5.5 \%$ & & & & \\
\hline Yes & 173 & $94.5 \%$ & & & & \\
\hline
\end{tabular}


The prevalence and severity of lung cancer patients' symptoms

The top three prevalent symptoms among participants were tiredness (99.5\%); distressed (98.9\%), and lack of appetite (97.3\%); whereas the rarest symptom was numbness (43.2\%). The average symptom severity was graded 4.0, which indicated symptoms disturbance with a mild-to-moderate level. Moreover, the top three severe symptoms experienced by the participants were fatigue, dry mouth, and shortness of breath. The prevalence and severity of symptoms were listed in Table 2.

Table 2 Prevalence and severity of Lung cancer patients' symptom

\begin{tabular}{lcl}
\hline & $\begin{array}{r}\text { Prevalence } \\
\mathbf{n}(\%)\end{array}$ & $\begin{array}{l}\text { Severity } \\
\text { Mean(SD) }\end{array}$ \\
\hline Fatigue & $182-99.5 \% \square$ & $6.24 \pm 1.70$ \\
Lack of appetite & $178 \square 97.3 \% \square$ & $4.86 \pm 2.11$ \\
Shortness of breath & $164 \square 89.6 \% \square$ & $4.88 \pm 2.52$ \\
Disturbed sleep & $175 \square 95.6 \% \square$ & $4.58 \pm 2.07$ \\
Distressed & $181 \square 98.9 \% \square$ & $4.26 \pm 1.96$ \\
Nausea & $150 \square 82.0 \% \square$ & $3.91 \pm 2.66$ \\
Dry mouth & $174 \square 95.1 \% \square$ & $4.96 \pm 2.01$ \\
Pain & $130 \square 71.0 \% \square$ & $3.78 \pm 3.01$ \\
Drowsiness & $79 \square 43.2 \% \square$ & $3.64 \pm 2.00$ \\
Vomiting & $138 \square 75.4 \% \square$ & $3.29 \pm 2.63$ \\
Sadness & $171 \square 94.4 \% \square$ & $3.56 \pm 2.16$ \\
Disturbed sleep & $142 \square 77.6 \% \square$ & $2.36 \pm 1.87$ \\
Numbness & $79 \square 43.2 \% \square$ & $1.39 \pm 2.03$ \\
\hline
\end{tabular}

\section{Symptom clusters}

The exploratory factor analysis with principal axis factoring was used to extract symptom clusters, and the Varian rotation was used to simplify the factor structure. In this study, variables with item-total correlation coefficient less than 0.4 were excluded because statistically a correlation with a lower than that will produce too many factors in factor analysis. Three distinct factors with Eigen values greater than 1.0 were retained, accounting for $69.4 \%$ of the total variance. The first factor, that is, gastrointestinal symptom cluster included nausea and vomiting. The second factor, fatigue-related cluster includes feeling fatigued, pain, and disturbed sleep. The third factor, emotional cluster, consisted of symptoms of sadness, and distress (Table 3).

Table 3 The Symptom Clusters in lung cancer $(n=183)$

\begin{tabular}{|c|c|c|c|c|}
\hline \multirow[t]{2}{*}{ Symptom clusters } & \multirow[t]{2}{*}{ Symptoms } & \multicolumn{3}{|c|}{ Factor Loading } \\
\hline & & Factor1 & Factor2 & Factor3 \\
\hline \multirow[t]{2}{*}{ Gastrointestinal } & nausea & 0.873 & & \\
\hline & vomiting & 0.887 & & \\
\hline Emotional & $\begin{array}{l}\text { distressed } \\
\text { sad }\end{array}$ & & $\begin{array}{l}0.724 \\
0.818\end{array}$ & \\
\hline \multirow[t]{2}{*}{ Fatigue related } & $\begin{array}{l}\text { disturbed sleep } \\
\text { disturbed sleep }\end{array}$ & & & $\begin{array}{l}0.826 \\
0.527\end{array}$ \\
\hline & pain & & & 0.450 \\
\hline
\end{tabular}


Relationship of symptom clusters to demographic and clinical information

For demographic variables, the gender differences were identified as statistically significant in each factor, which indicated that females reported statistically higher troubling symptoms than males. Education differences among the three symptom clusters were statistically significant. Chronic illnesses were significantly related to gastrointestinal symptom clusters as shown in table 4.

Table 4 The correlations among demographic characteristics

\begin{tabular}{lllllll}
\hline Variable & $\begin{array}{l}\text { Factor1 } \\
t / F\end{array}$ & $\mathrm{P}$ & $\begin{array}{l}\text { Factor2 } \\
t / F\end{array}$ & $\mathrm{P}$ & $\begin{array}{l}\text { Factor3 } \\
t / F\end{array}$ & $\mathrm{P}$ \\
\hline Gender & -2.99 & .01 & -2.32 & .02 & -2.18 & .03 \\
Stage of disease & 5.73 & .001 & 5.94 & .001 & 4.81 & .001 \\
chronic diseases & 2.45 & .04 & & & & \\
\hline
\end{tabular}

\section{Relationship between symptom clusters and quality of life}

The results demonstrated the descriptive statistics for the FACT-L total and its subscales. The highest HRQOL score for the total sample was the lung cancer subscale dimension, followed by the social dimension.
The lowest score was the role functioning dimension, followed by the emotional dimension. Pearson correlations were computed between the symptom cluster scale scores and the quality-of-life measurements and the results are in table 5 .

Table 5 Worse physical quality of life was associated with higher symptom bother from all symptom clusters

\begin{tabular}{lcccc}
\multicolumn{1}{c}{ Variables } & M(SD) & gastrointestinal & $\begin{array}{c}\text { Symptom } \\
\text { Clusters } \\
\text { emotional }\end{array}$ & Fatigue related \\
\hline FACT-L & $14.38 \pm 4.72$ & -.655 & -.697 & -.749 \\
Physical well-being & $19.28 \pm 3.24$ & -.178 & -.302 & -.257 \\
Social/family well-being & $13.20 \pm 4.37$ & -.358 & -.805 & -.617 \\
Emotional well-being & $9.70 \pm 5.30$ & -.411 & -.749 & -.696 \\
Functional well-being & $20.89 \pm 5.55$ & -.332 & -.508 & -.485 \\
Lung cancer subscale & & & & \\
\hline
\end{tabular}

\section{Discussion}

The results of this study have provided important insights into the symptom experiences and the relationship between symptom clusters and quality of life in patients with lung cancer in mainland China. Lung cancer remains the leading cause of cancer death in mainland China. However, studies describing the experiences of patients living with lung cancer in mainland China are limited. To our knowledge, this is the first study in mainland China, to explore symptom clusters as well as test the relationship between symptom clusters and quality of life in a homogeneous group of lung cancer patients.

Tiredness, distress, and lack of appetite were the most prevalent symptoms in this study. This result was similar to that from previous studies[16-18]. Concerning severity of symptoms, our result showed fatigue, dry mouth, and shortness of breath as the most severe, was similar to Semiha Akin 's [19] findings. The results of this study indicate lung cancer patients in mainland China suffered from a severe level of symptom distress similar to what is experienced in Europe and north America. Three factors were identified this study. Gastrointestinal symptom cluster included nausea and vomiting, which was similar to the research of Wang, SY et al[20]. Gastrointestinal symptom cluster probably resulted from the side effects of chemotherapy. Fatigue-related cluster composed of feeling fatigued, pain, and disturbed sleep. These sickness behaviors were considered to be mediated by proinflammatory cytokines[21,22]. Emotional cluster which was a more psychologically related cluster consisted of symptoms of sad, and distressed. Historically, cancer symptom research had focused on a single symptom at a time, but now, we were aware that the patients with lung cancer often have multiple concurrent symptoms and may exacerbate one another. Furthermore, assessing possible symptom grouping simultaneously, systematically and comprehensively and therefore treated; interest in the effectively symptom management of cancer patients should pay more attentions to symptom clusters instead of only to individual symptoms. 
It was found to be correlated with all symptom clusters in this study were gender and education. Weakness, psychological vulnerability was the nature of female patients leading to more symptom burden than male. The difference caused by education was similar to the previous research[23]. Patients who received high education will have early recognition of cancer and seek treatment actively. The weak correlations between symptom cluster and chronic disease might be explained by two reasons. Firstly, the symptoms of chronic disease itself will contribute to cancer symptom cluster. Secondly, lung cancer patients with chronic diseases produce more economic and psychological burden than patients without.

In this study, we found lung cancer patients had low QOL scores. The functioning subscale scores were the lowest, while the family subscale scores were the highest. The current study found a negative relationship between symptom clusters and QOL scores. Generally, almost all three symptom clusters were negatively correlated with PWB, EWB, FWB, SWB, and overall QOL. In this study, physical quality of life was highly correlated with all symptom clusters. Another particularly strong relationship was between the emotional cluster and emotional well-being in quality of life. This finding was consistent with other studies[24-26].

Overall QOL was important for patients with lung cancer. Therefore, symptom and QOL assessments were vital for the evaluation of the efficacy of cancer treatments. Patients with cancer experienced different levels of symptom distress according to disease and treatment. According to our finding, symptom management should be taken into account in order to decrease the negative effects of symptom clusters on QOL. Further studies should include a larger sample to more fully describe the potential interactive effects of the dependent variables on QOL.

\section{Limitations and methodological issues:}

Firstly, the MDASI was a relatively short instrument that assessed only13 symptoms. As we know, using different tools of symptoms may result in different symptom clusters. We therefore suggested a more comprehensive tool should be used to assess symptoms when exploring the phenomenon of symptom clusters. Secondly, the current study of symptom clusters had been conducted by cross-sectional design. A future study was needed to explore long-term changes of symptom clusters and its association with physical, psychological, and clinical variables.

\section{Conclusions}

Lung cancer patients receiving chemotherapy suffered from numerous symptoms simultaneously, due to the disease itself as well as treatment. The results of this study have provide understanding of symptom clusters which could help clinicians develop more comprehensive and useful assessment tools as well as more effective symptom management strategies to improve the quality of life for patients with lung cancer in china.

\section{Conflict of interest}

The authors have no conflict of interest to declare.

\section{References}

1.http://www.phsciencedata.cn/Share / advancedSearchDataSet.jsp.[EB/OL].2012/9/30.

2. Gift AG, Jablonski A, Stommel M, Given CW. Symptom clusters in elderly patients with lung cancer. Oncol Nurs Forum. 2004; 31(2), 202-212.

3. Dodd MJ, Miaskowski C, Paul SM. Symptom clusters and their effect on the functional status of patients with cancer. Oncol Nurs Forum. 2001; 28(3), 465-470.

4. Fan G, Filipczak L, Chow E. Symptom clusters in cancer patients: a review of the literature. Curr Oncol. 2007; 14(5), 173-179.

5. Kim HJ, McGuire DB, Tulman L, Barsevick AM. Symptom clusters: concept analysis and clinical implications for cancer nursing. Cancer Nurs. 2005; 28(4), 270-282, 283-284.

6. Given B, Given CW, McCorkle R, Kozachik S, Cimprich B, Rahbar $\mathrm{MH}$, et al. Pain and fatigue management: results of a nursing randomized clinical trial. Oncol Nurs Forum. 2002; 29(6), 949-956.

7. Given CW, Given B, Azzouz F, Kozachik S, Stommel M. Predictors of pain and fatigue in the year following diagnosis among elderly cancer patients. J Pain Symptom Manage. 2001; 21(6), 456-466.

8. Lenz ER, Pugh LC, Milligan RA, Gift A, Suppe F. The middle-range theory of unpleasant symptoms: an update. ANS Adv Nurs Sci. 1997; 19(3), 14-27.

9. Chan CW, Richardson A, Richardson JA. study to assess the existence of the symptom cluster of breathlessness, fatigue and anxiety in patients with advanced lung cancer. Eur J Oncol Nurs. 2005; 9(4), 325-333.

10. Fox SW, Lyon DE. Symptom clusters and quality of life in survivors of lung cancer. Oncol Nurs Forum. 2006; 33(5), 931-936.

11. Gift AG, Jablonski A, Stommel M, Given CW. Symptom clusters in elderly patients with lung cancer. 
Oncol Nurs Forum. 2004; 31(2), 202-212.

12. Hoffman AJ, Given BA, von Eye A, Gift AG, Given CW. Relationships among pain, fatigue, insomnia, and gender in persons with lung cancer. Oncol Nurs Forum. 2007; 34(4), 785-792.

13. Lenz ER, Pugh LC, Milligan RA, Gift A, Suppe F. The middle-range theory of unpleasant symptoms: an update. ANS Adv Nurs Sci. 1997; 19(3), 14-27.

14. Salvo N, Hadi S, Napolskikh J, Goh P, Sinclair E, Chow E. Quality of life measurement in cancer patients receiving palliative radiotherapy for symptomatic lung cancer: a literature review. Curr Oncol. 2008; 16(2), 1628.

15. Wang CH, Zhang CZ, et al. (2001)The Research on Measurement for Quality of life in Patients with Lung Cancer J]. Chinese cancer, 10(2):83-85.

16. Cooley ME. Symptoms in adults with lung cancer. A systematic research review. J Pain Symptom Manage. 2000; 19(2), 137-153.

17. Gift AG, Jablonski A, Stommel M, Given CW. Symptom clusters in elderly patients with lung cancer. Oncol Nurs Forum. 2004; 31(2), 202-212.

18. Wang SY, Tsai CM, Chen BC, Lin CH, Lin CC. Symptom clusters and relationships to symptom interference with daily life in Taiwanese lung cancer patients. J Pain Symptom Manage. 2008; 35(3), 258266.

19. Akin S, Can G, Aydiner A, Ozdilli K, Durna Z.
Quality of life, symptom experience and distress of lung cancer patients undergoing chemotherapy. Eur J Oncol Nurs. 2010; 14(5), 400-409.

20. Wang SY, Tsai CM, Chen BC, Lin CH, Lin CC. Symptom clusters and relationships to symptom interference with daily life in Taiwanese lung cancer patients. J Pain Symptom Manage. 2008; 35(3), 258266.

21. Cleeland CS, Bennett GJ. Dantzer R, Dougherty PM, Dunn AJ. Meyers CA. et al. Are the symptoms of cancer and cancer treatment due to a shared biologic mechanism? A cytokine-immunologic model of cancer symptoms. Cancer. 2003; 97(11), 2919-2925.

22. Dantzer R. Cytokine-induced sickness behavior: mechanisms and implications. Ann N Y Acad Sci. 2001; 933, 222-234.

23. Roiland RA, Heidrich SM. Symptom clusters and quality of life in older adult breast cancer survivors. Oncol Nurs Forum. 2011; 38(6), 672-680.

24. Dodd MJ, Miaskowski C, Paul SM. Symptom clusters and their effect on the functional status of patients with cancer. Oncol Nurs Forum.2001; 28(3), 465-470.

25. Fox SW, Lyon DE. Symptom clusters and quality of life in survivors of lung cancer. Oncol Nurs Forum. 2006; 33(5), 931-936.

26. Miaskowski C. Symptom clusters: establishing the link between clinical practice and symptom management research. Support Care Cancer. 2006; 14(8), 792-794. 